\title{
Initial experience of robotic single port plus one $(S P+1)$ distal pancreatectomy using the da Vinci SP system: A report of three cases with video
}

\author{
Young-Dong YU*, Hye-Sung JO, Dong-Sik KIM
}

Department of Surgery, Korea University Anam Hospital, Seoul, Korea

Introduction: With the advances of laparoscopic techniques, many efforts to reduce the number of the trocar site in abdominal surgery has been made. However, laparoscopic single-site distal pancreatectomy is regarded as difficult and technically demanding. Robotic surgical system was introduced to overcome the limitation of conventional laparoscopic surgery. The da Vinci surgical system released its new pure single-port platform, the da Vinci SP, offering improvements and refinements for established robotic single-site procedures. We report our initial experience of robotic single port plus one port $(\mathrm{SP}+1)$ distal pancreatectomy using the da Vinci SP system. To our knowledge, this is the first report in Korea using the da Vinci SP system for distal pancreatectomy.

Methods: We reviewed the medical records of three patients in whom robotic single port plus one port $(\mathrm{SP}+1)$ distal pancreatectomy was carried out. Robotic distal pancreatectomy was performed using the da Vinci SP system with one additional port. An additional robotic 12-mm-port was placed on the left side of the da Vinci SP system and was used for applying energy device such as Thunderbeat and endo-GIA stapling.

Results: The median age was 70 years (range 65-77). Pathological diagnosis included pancreas serous cystadenoma, neuroendocrine tumor and unsuspected pancreatic cancer. Median operation time was 245 minutes (range 135-265 minutes). All patients underwent combined splenectomy. There was no clinically relevant postoperative pancreatic fistula. Length of hospital stay was median 10 days after surgery.

Conclusions: Robotic single port distal pancreatectomy using the da Vinci SP system is safe and feasible with acceptable perioperative outcomes. 